\title{
Stage III Prostate Cancer AJCC v6
}

National Cancer Institute

\section{Source}

National Cancer Institute. Stage III Prostate Cancer A/CC v6. NCI Thesaurus. Code C7551.

Stage III includes: T3, N0, M0, Any G. T3: Tumor extends through the prostatic capsule.

T3a: Extracapsular extension (unilateral or bilateral). T3b: Tumor invades seminal vesicle(s). N0: No regional lymph node metastasis. M0: No distant metastasis. (AJCC 6th ed.) - 2003 